\title{
Estudio de la importancia de la educación superior a distancia en el marco de la educación permanente
}

\author{
Antonio Alfaro Fernández ${ }^{1}$ \\ Recibido: 16-03-2015 - Aceptado: 03-09-2015
}

\begin{abstract}
Resumen
La educación se ha configurado como un nuevo estilo de vida que no se limita al aprendizaje formal que se lleva a cabo en la infancia y la juventud, el convencimiento de que la educación es un proceso que dura toda la vida es una realidad. La igualdad de oportunidades y el derecho a la educación a lo largo de la vida se convirtieron en el siglo XX en principios que unidos al derecho universal a la educación han transformado el panorama educativo imperante hasta el momento. El trabajo pretende poner de manifiesto la importancia y necesidad de la educación permanente en el actual contexto socioeconómico. Tanto por los requerimientos planteados por el cambiante panorama laboral, como por lo imperativo de actualizar los conocimientos y responder a las nuevas demandas de la sociedad, entre las que podemos destacar el bienestar social que exige una adecuada educación para el ocio. Asimismo, se plantea el papel relevante que juega la educación a distancia dentro del paradigma de la educación permanente, centrados en el ámbito de la educación superior. De forma complementaria, se presentan los resultados de una investigación cuyo trabajo de campo se realizó durante el curso 2011-12, en el que se analiza el perfil del alumnado de educación superior a distancia a través de la información recogida mediante un cuestionario diseñado para este trabajo. A través de más de 1.100 encuestas se ha podido conocer datos personales, familiares, laborales y académicos de las personas participantes en el estudio. Para finalizar y tras el análisis de los resultados se presentan las conclusiones alcanzadas en esta investigación.
\end{abstract}

Palabras clave: Educación, educación permanente, educación de adultos, educación a distancia, educación superior, estudiantes.

\footnotetext{
${ }^{1}$ Licenciado en Pedagogía y doctor por la Universidad Nacional de Educación a Distancia -UNED. Tutor en Centro Asociado de la UNED de Albacete, y profesor asociado en la Facultad de Ciencias de la Educación y Humanidades de la Universidad de CastillaLa Mancha, España; antonio.alfaro@uclm.es
} 
BÚSQUEDA - Julio / Diciembre de 2015 - No. 15 (7 - 17)

\title{
Study of the importance of higher education distantly in the frame of the lifelong education
}

\begin{abstract}
Education has been set up as a new lifestyle that is not limited to formal learning that takes place in childhood and youth, the belief that education is a process that lasts a lifetime is a reality. Equal opportunity and the right to education throughout life have become the early twentieth century that united the universal right to education have transformed the educational landscape prevailing so far. The work aims to highlight the importance and need for continuing education in the current economic context. Both the needs raised by the changing employment landscape as the need to update our knowledge and meet the new demands of society, among which we highlight the social welfare demanded adequate education for leisure. It also highlights the important role played by distance education within the paradigm of lifelong learning, focusing on the field of higher education. To complement this, we present the results of an investigation whose field work was conducted during the year 2011-12, which analyzes the profile of students in higher distance education through information collected through a questionnaire designed for this job. Through more than 1,100 surveys have been able to know personal details, family, work and academic people in the study. Finally and after analysis of the results are presented the conclusions reached in this research.
\end{abstract}

Keywords: Education, lifelong education, adult education, distance learning, higher education, students.

\section{Introducción}

Las futuras prioridades del desarrollo de la educación deben reflejar estos cambios importantes que han ocurrido y seguirán ocurriendo. Es necesario estudiar de qué modo deben adaptarse los sistemas educativos para responder satisfactoriamente a los desafíos contemporáneos y contribuir al desarrollo sostenible y la paz. Esto requiere repensar el tipo de conocimientos, habilidades y competencias que se requieren para el futuro, así como la clase de procesos educativos y de aprendizaje que podrían facilitarlos y, en última instancia, qué políticas y reformas de la educación para ese cambio se requieren. (UNESCO, 2015).

La sociedad está atravesando un momento de cambio e incertidumbre motivado por la crisis económica y social que azota el mundo en los últimos años. La situación económica y las terribles tasas de desempleo hacen necesaria una política educativa que posibilite la alternancia entre educación y formación con el trabajo cuando la persona lo necesite, con las máximas garantías de flexibilidad y con una oferta educativa que responda a las demandas reales de la sociedad y de las personas.

Ante este panorama cobra más fuerza y relevancia, si cabe, el principio de educación permanente.

Los objetivos de este trabajo son reconocer la importancia del principio de educación permanente, resaltar el papel que juega la educación a distancia dentro de este paradigma e identificar el perfil sociológico del alumnado de educación superior en una institución de educación a distancia.

La educación durante toda la vida se presenta como una de las llaves de acceso al siglo XXI. Esta noción va más allá de la distinción tradicional entre educación básica y educación permanente, y responde al reto de un mundo que cambia rápidamente. Pero esta afirmación no es nueva, puesto que en anteriores informes sobre educación ya se destacaba la necesidad de volver a la escuela para poder afrontar las novedades que surgen en la vida privada y en la vida profesional. Esta necesidad persiste, incluso se ha acentuado, 
y la única forma de satisfacerla es que todos aprendamos a aprender (Delors, 1996).

La XIX Conferencia General de la UNESCO (Nairobi, 1976) define la educación permanente como "un proyecto global encaminado tanto a reestructurar el sistema educativo existente como a desarrollar todas las posibilidades de formación fuera del sistema educativo", en este proyecto:

- La persona es el agente de su propia educación por medio de la interacción permanente de sus acciones y su reflexión.

- Debeabarcar todas las dimensiones de la vida y no limitarse al período de escolarización, todas las ramas del saber y todos los conocimientos prácticos adquiridos por cualquier medio, así como contribuir al desarrollo de la personalidad.

- Los procesos educativos seguidos a lo largo de la vida de niños, jóvenes y adultos, deben ser considerados como un todo.

La educación permanente se ha convertido en un nuevo paradigma educativo que se contrapone al que tradicionalmente se ha realizado en la escuela. La educación como nuevo estilo de vida está orientada hacia la persona y no hacia la institución. Rechaza la idea de que el aprendizaje formal es solo para los jóvenes y la reemplaza por el convencimiento de que la educación es un proceso que dura toda la vida (MartínezSalanova, 2012).

Aunque suele presentarse como algo reciente, la idea de que la educación, la enseñanza y el aprendizaje no están limitados a un período de la vida es bastante antigua, así como el reconocimiento de todas las posibilidades educativas que ofrece la vida (Bajo, 2009). Este autor expone en su obra citas que manifiestan que Sófocles y Platón hace más de dos mil años ya pensaban que la educación a lo largo de la vida era necesaria. Ya en el siglo XVII Comenius escribe "toda la vida es una escuela para los hombres, del nacimiento a la tumba".
En el siglo XIX surgen movimientos que apoyaban y promocionaban el aprendizaje de adultos, fundamentalmente para la clase trabajadora industrial que se desarrollaba fuera del entorno escolar.

Pero sin duda, es en el siglo XX cuando el concepto de educación permanente alcanza mayor popularidad y se le ha otorgado mayor valor.

Este concepto parece más relacionado en la actualidad a la adecuación de las necesidades socioeconómicas de nuestra sociedad que a los movimientos abanderados por Illich, Reimer y Goodman quienes lo planteaban como alternativa a los sistemas educativos de la segunda mitad del siglo XX.

En el año 2000, el desarrollo de una educación y formación permanente para todos se constituyó en uno de los elementos claves para conseguir el objetivo de que la Unión Europea se convirtiese en una economía basada en el conocimiento, la más dinámica del mundo.

La Resolución del Consejo de la Unión Europea de 27 de junio de 2002, señala que la educación permanente tiene que comprender el aprendizaje, desde la etapa preescolar hasta después de la jubilación, incluyendo todo el espectro de aprendizaje formal, no formal o informal. La educación permanente constituye una política esencial para el desarrollo de la ciudadanía, la cohesión social y el empleo.

Caride, Lorenzo y Rodríguez (2012), afirman que la educación es una misión que nos sitúa más allá de la posibilidad de aprender durante toda la vida, al requerir que incluso se haga en todo momento. Gimeno Sacristán (2008), expone "el tiempo de educarse es todo el tiempo, en cualquier oportunidad, sobre temas de muy diverso tipo". De ahí que no deba interpretarse exclusivamente como un tiempo pautado por los horarios escolares, sino por principios, valores e iniciativas que abran las fronteras de la educación a las anchas avenidas de lo cultural y lo social, cotidianamente, desde la infancia hasta la vejez. 
BÚSQUEDA - Julio / Diciembre de 2015 - No. 15 (7 - 17)

En los últimos tiempos, ya sea por el impacto de la Ilamada Sociedad de la Información y del Conocimiento o sea, simplemente, porque las relaciones sociolaborales de las personas deben readaptarse a nuevas situaciones empresariales y personales, se está asistiendo a un cambio de hábitos de los individuos que se están reflejando también en los procesos de formación. Así, y especialmente a medida que la edad de los estudiantes aumenta y, con ella, las responsabilidades a que están sujetos, la necesidad de ofrecer sistemas de formación que superen los obstáculos generados por los desplazamientos o por la falta de tiempo para asistir a las clases, se hace cada vez más evidente (Sangrá, 2002).

Mora (2006), considera necesario hacer frente a situaciones sociales que obligan a una mayor demanda educativa, que precisa una intensa capacitación para asumir y llevar a la práctica las nuevas actividades que implica el creciente desarrollo tecnológico, así como contar con competencias profesionales mejor calificadas, que como resultado de los cambios acelerados, se convierten rápidamente en obsoletas. Para atender esta creciente demanda de estudios, es necesario contar con instituciones, que, sin perder su tradicional carácter académico y científico, tengan la posibilidad de dar una respuesta flexible y clara a esta nueva modalidad de alumnado, que necesita una constante información y puesta al día y que encuentra serias dificultades para asistir regularmente a los centros de enseñanza universitarios convencionales.

Por todo lo expuesto, se considera que la educación a distancia puede desempeñar un papel preponderante en la resolución de las demandas de educación permanente planteadas por la sociedad. Su desarrollo va ligado a los grandes avances de la modernidad. En primer lugar, a las necesidades generadas por la industrialización y después a la aparición de las tecnologías de la información y la comunicación que plantearon la exigencia y la posibilidad de extender la educación permanente a toda la población.

\section{Educación a distancia, antecedentes y} desarrollo

La educación a distancia juega un importante papel dentro del paradigma de la educación permanente, esta modalidad está proporcionando a los adultos una segunda oportunidad de estudios superiores y está ayudando a quienes tienen limitaciones de tiempo, distancia, trabajo o limitaciones físicas, a actualizar los conocimientos requeridos en sus trabajos (Álvarez, 1998).

Castells (1997), afirmaba que hasta hace relativamente poco, la educación a distancia era observada como una educación de carácter compensatorio, a la cual estaban "condenadas" aquellas personas que no tenían la posibilidad de asistir a situaciones de educación presencial; la emergencia del uso social de las tecnologías de la información y la comunicación, conjuntamente a la conceptualización de la educación como un proceso que se extiende a lo largo de la vida (Delors, 1996), han hecho que la educación a distancia pueda considerarse en estos momentos, y en algunos casos, como una alternativa real a la educación presencial (Sangrá, 2002).

El número de estudiantes universitarios en España de todas las etapas fue durante el curso 2011-12 de 1.582.714 alumnos, un 2,3\% más que en el 2010-11, según datos facilitados por el Ministerio de Educación, Cultura y Deporte. En las universidades públicas durante este curso han estudiado 1.469.653 alumnos en la primera etapa universitaria (grado y primer y segundo ciclo), lo que supone un incremento del 1,7\% respecto al anterior, mientras que ha descendido un $2 \%$ en las universidades privadas, con un total de 172.442 alumnos. El crecimiento más significativo lo ha registrado la Universidad Nacional de Educación a Distancia (UNED), con un aumento del 14,7\%, con 179.781 estudiantes en esta primera etapa universitaria.

En España existen cinco universidades a distancia con la siguiente distribución de matriculación en el curso 2011-12 (ver tabla 1 en anexos). 
Antonio Alfaro F. - Estudio de la importancia de la educación superior a distancia

Una vez resaltada la importancia de la educación a distancia en el ámbito de la educación superior en territorio español, se hará un repaso sobre el fenómeno de la educación a distancia a lo largo de la historia.

Si se buscase el origen de la educación a distancia sería válido remontarse hasta las civilizaciones más antiguas. Graf (1980) cita las civilizaciones sumerias y egipcias, y destaca la epistolografía griega que alcanzó un alto nivel en las cartas de contenido instructivo.

El inicio de la educación a distancia, tal como la entendemos hoy, se inicia con el desarrollo de los sistemas nacionales de correos, que se sitúan en 1680 con el penny post. Battenberg (1971) cita como primer dato un anuncio de Caleb Philipps, profesor de Short Hand, en la Gaceta de Boston ofreciendo material de enseñanza y tutorías por correspondencia el 20 de marzo de 1728.

En el curso académico 1940-41 existían en la URSS unos 200.000 alumnos inscritos en estudios de educación superior por correspondencia (García, 2002). Este mismo autor cita la Open University Británica como institución pionera de lo que hoy se entiende como educación superior a distancia. En 1972, en España se creó la Universidad Nacional de Educación a Distancia (UNED). Tras la creación de estas dos universidades nacieron las siguientes instituciones en Europa: Fernuniverität de Hagen, Alemania (1974); Open Universiteit de Holanda (1980); National Distance Education Center de Irlanda (1982); Swedish Association for Distance Education de Suecia (1984); Studiecentrum Open Hoger Onderwijs, en Bélgica (1987); Federation Interuniversitaire de L'Enseignement a Distance de Francia (1987); Universidad Aberta de Portugal (1988); The Norwegian Executive Board for Distance Education at University and College Level de Noruega (1990).

En Heerlen (Holanda) se creó la European Association of Distance Teaching Universities en 1987. Esta institución tiene como objetivo fomentar la educación superior a distancia en Europa.
Dentro del desarrollo que la educación a distancia ha experimentado, es posible distinguir las siguientes generaciones (Garrison, 1985):

- La enseñanza por correspondencia.

- La enseñanza multimedia.

- La enseñanza telemática.

Taylor (1995), añade una cuarta generación: la enseñanza vía Internet. Taylor en 1999, propuso una quinta generación: aprendizaje flexible inteligente.

García (2002), señala los factores que propician el desarrollo de la educación a distancia:

- Los avances sociopolíticos.

- La necesidad de aprender a lo largo de la vida.

- La carestía de los sistemas convencionales.

- Los avances en el ámbito de las ciencias de la educación.

- Las transformaciones tecnológicas.

Después del análisis de gran cantidad de definiciones, que ponen de manifiesto la dificultad de definir el concepto de educación a distancia, se propone la siguiente:

La enseñanza a distancia es un sistema tecnológico de comunicación bidireccional (multidireccional), que puede ser masivo, basado en la acción sistemática y conjunta de recursos didácticos y el apoyo de una organización y tutoría, que, separados físicamente de los estudiantes, propician en estos un aprendizaje independiente (cooperativo). (García, 2002, p. 32).

En esta definición se pueden identificar las características que definen a la educación a distancia, y que surgen del estudio comparativo de la definición de este concepto por distintos autores:

- Separación profesor-alumno.

- Utilización de medios técnicos.

- Organización de apoyo-tutoría.

- Aprendizaje independiente y flexible.

- Comunicación bidireccional. 
BÚSQUEDA - Julio / Diciembre de 2015 - No. 15 (7 - 17)

- Enfoque tecnológico.

- Comunicación masiva.

- Procedimiento industrial.

El calificativo de educación a distancia referido a una modalidad educativa resulta cada vez más difícil de aplicar. El empleo progresivo de las nuevas tecnologías de la comunicación hace muchas veces inadecuado el término «a distancia», cuando permiten la comunicación prácticamente en tiempo real y pueden crear entornos virtuales de aprendizaje que rompen la distancia física indicada (Sarramona, 2001).

García (1986, 1990), enumera los objetivos generales de las instituciones de educación a distancia:

- Democratizar el acceso a la educación.

- Propiciar un aprendizaje autónomo y ligado a la experiencia.

- Impartir una enseñanza innovadora y de calidad.

- Fomentar la educación permanente.

- Reducir los costes.

Jorquera (1999), afirma que la educación a distancia desde el punto de vista de los procesos curriculares, acredita la experiencia adquirida y los conocimientos previos del estudiante, lo que permite un aprendizaje realmente significativo. El aporte de las teorías constructivistas encuentra en la educación a distancia un excelente campo de aplicación. Este es un tipo de educación académicamente exigente, especialmente por las características que los usuarios deben desarrollar como pre-requisitos: capacidades de lectura comprensiva, de identificación y solución de problemas, de análisis y de crítica, habilidad para investigar y comunicar adecuadamente los resultados. A pesar de que el estudio es individual, no se descarta el trabajo en pequeños grupos, aun más, es recomendado. "La modalidad de trabajo grupal y el hecho de resolver una tarea en cooperación con otros puede redundar en beneficios para la motivación y el aprendizaje" (Chiecher, Paoloni y Ficco, 2014).
Como factores de peso en el rendimiento del alumnado de educación a distancia aparecen la motivación y la autodisciplina, ya que las características de la modalidad, que implica un menor grado de control directo sobre el alumnado que en la formación presencial, exige un mayor esfuerzo personal al estudiante, quien ya no responderá a obligaciones marcadas en forma externa, sino que deberá construir su propio esquema de objetivos y exigencias de logro en el marco de las pautas ofrecidas por el sistema, pero desde un enfoque absolutamente subjetivo (Padula, 2002).

Una vez contextualizado el paradigma de educación permanente y planteada la importancia de la educación a distancia en el mismo, se presenta un estudio experimental sobre el perfil del alumnado de educación superior a distancia.

Para desarrollar la parte experimental de este trabajo se decidió utilizar como muestra el alumnado de la Universidad Nacional de Educación a Distancia (UNED), más concretamente del Centro Asociado de Albacete. La UNED es la primera y más importante institución de educación superior a distancia en territorio español, así como la que plantea una de las ofertas más amplias y ambiciosas en el marco de la educación permanente.

En la UNED, en el curso 2011/12 se completó la oferta académica de los 27 títulos de grado que constituyen el mapa básico de grados de la UNED, con lo que se adecuaba la oferta educativa al espacio europeo de educación superior, que junto con los títulos en extinción, suponen un total de 179.781 estudiantes. En relación con los másteres oficiales se han ofertado 49 títulos con 5.814 estudiantes. A los que hay que sumar los 1.100 integrantes de los programas de doctorado.

Las enseñanzas correspondientes a los cursos de acceso directo para mayores de 25 años y 45 años siguen teniendo una gran demanda social, con una matrícula confirmada de 25.185 alumnos.

En lo relativo a las enseñanzas de formación permanente, en el pasado curso contó con 32.181 
estudiantes, mientras que la cifra de matrícula en el Centro de Idiomas a Distancia (CUID) ascendió a los 12.427 estudiantes; lo que supone un 19\% de incremento con respecto al año anterior.

En total, 239 mil estudiantes se matricularon el curso referido en la UNED.

Los 62 Centros Asociados que configuran la UNED, además de las tutorías presenciales y telemáticas, impartidas gracias a los casi 7.000 profesores-tutores, son auténticos enclaves dinamizadores de sus ciudades a través de sus bibliotecas, de los cursos de verano que siguen incrementando año tras año (8.276 en el año 2012) y las casi mil actividades de extensión universitaria. Estos centros son un ejemplo de colaboración y permiten acercar aún más el servicio universitario a los ciudadanos.

La segunda de las grandes líneas del plan estratégico de la UNED es la formación a lo largo de la vida; y es que una de las funciones primordiales de esta universidad es contribuir a la configuración de la sociedad del conocimiento, en su más amplia acepción. El plan de formación permanente en la UNED está constituido por un total de 582 cursos de formación continua, de entre los cuales cabe señalar el curso "Acredítate", que ha formado a más de 3.000 asesores y orientadores en evaluación de competencias adquiridas por la experiencia laboral.

\section{Metodología}

En este estudio, en función del problema de investigación planteado y de los objetivos presentados, el enfoque metodológico que se ha seguido responde a un diseño cuantitativo y será predominantemente descriptivo, a partir de los datos derivados del uso del cuestionario.

\section{Objetivos}

Los objetivos establecidos para la parte experimental del trabajo, son los siguientes:

- Conocer el perfil sociológico del alumnado de educación superior a distancia.
- Identificar las condiciones laborales de los estudiantes.

- Conocer los motivos que mueven a los estudiantes para cursar estudios universitarios y la modalidad de acceso a los mismos.

\section{Muestra}

Este trabajo se realizó a lo largo del curso académico 2011-12 y la población objeto estudio estuvo constituida por el alumnado de las titulaciones ofertadas en el Centro Asociado de la UNED de Albacete. Por lo tanto, el alumnado matriculado durante este curso $(n=2.294)$.

Fue informado adecuadamente del carácter voluntario y anónimo de su participación en el estudio, así como de los objetivos del mismo.

Las encuestas se le presentaron mediante la modalidad on-line; la muestra responde a un modelo incidental, y la muestra aceptante está compuesta por las encuestas contestadas por el alumnado que accedió a participar en el estudio. La muestra productora de datos está compuesta por las 1.170 encuestas contestadas correctamente.

\section{Instrumentos}

Para la realización de esta investigación, el instrumento utilizado ha sido el cuestionario estructurado, pues posee ventajas importantes como su capacidad para llegar a amplios grupos de personas, para recoger gran cantidad de información y su facilidad para analizarla y tratarla de forma detallada y hasta exhaustiva (Pérez, 2006).

La razón para construir el cuestionario ha sido la imposibilidad de encontrar un instrumento que recogiera las especiales características del alumnado de la UNED.

Como paso previo a la construcción del cuestionario se pensó qué tipo de información se quería recoger y para qué, luego se buscaron otros cuestionarios que hubieran sido diseñados para usos similares. Se realizó un primer borrador que fue sometido al juicio de un grupo de expertos. Se reelaboró el cuestionario recogiendo 
BÚSQUEDA - Julio / Diciembre de 2015 - No. 15 (7 - 17)

sus aportaciones y se hizo una prueba piloto con una muestra de alumnas y alumnos para conocer si era bien entendido y el tiempo necesario para contestarlo.

El cuestionario para recoger la opinión del alumnado constaba de siete ítems, dentro de cada uno el encuestado debía señalar la opción que se ajustaba a su perfil.

La redacción de los ítems se hizo teniendo en cuenta las consideraciones técnicas habituales en estos casos: claridad, facilidad de interpretación, lenguaje conocido, ausencia de preguntas ambiguas, etc.

La validación del contenido se contrastó mediante el juicio de expertos, y se realizó en función de los siguientes criterios:

- La univocidad, se evalúan el aspecto formal y de expresión del ítem.

- La pertinencia, en definitiva, si el ítem es pertinente o no.

- El grado de importancia del ítem respecto al objeto evaluado.

- Extensión del cuestionario en su conjunto.

\section{Resultados}

Para estudiar la información recogida se realizó un análisis de frecuencias. A continuación, se presentan los resultados obtenidos en cada uno de los ítems.

El sexo de los encuestados se distribuía según los datos de la siguiente tabla (ver tabla 2 en anexos).

Se puede comprobar que el sexo del alumnado de educación superior a distancia sigue el patrón de la universidad presencial, donde la mujer es mayoría desde hace décadas.
La edad del alumnado encuestado se detalla a continuación (ver tabla 3 en anexos).

A pesar de que la edad se distribuya en una amplia de horquilla de edad, se puede comprobar que más del cincuenta por ciento de los encuestados se encuadra en la franja comprendida entre los 31 y los 50 años. Esta circunstancia se puede justificar porque se trata de la segunda carrera o porque se trata de personas que no tuvieron oportunidad de estudiar por diversos motivos en su juventud y eligen la educación a distancia para ello.

En lo referente a los alumnos con hijos encontramos los siguientes datos (ver tabla 4 en anexos).

Es de destacar que más del cincuenta por ciento de los encuestados son padre o madre, hay que valorar el esfuerzo que supone cursar estudios superiores cuando existen obligaciones familiares que exigen gran sacrificio para compatibilizar y conciliar la vida familiar y los estudios.

El número de alumnos que trabajaban y estudiaban es el siguiente (ver tabla 5 en anexos).

Los datos revelan que un gran porcentaje del alumnado compatibiliza trabajo y estudios, lo que pone de manifiesto las dificultades que deben superar los alumnos de educación a distancia. Asimismo, se justifica la elección de esta modalidad debido a la limitación de tiempo para asistir a clases de manera presencial.

La dedicación semanal al trabajo se detalla en la siguiente tabla (ver tabla 6 en anexos).

A pesar de compatibilizar trabajo y estudios una gran mayoría de encuestados cumple con una jornada laboral completa. Se les preguntó si trabajaban en algún ámbito relacionado con la carrera estudiada, en la siguiente tabla se reflejan los resultados obtenidos (ver tabla 7 en anexos).

Respecto al ámbito laboral de los encuestados que trabajan, se puede observar que más del cincuenta por ciento trabaja en un 
campo relacionado con la carrera estudiada, lo que puede interpretarse como la existencia de motivación por promocionarse profesionalmente. El hecho del considerable porcentaje de personas que estudia carreras no relacionadas con su ámbito laboral revela que persiguen un cambio de su actividad profesional o simplemente que estudian por el placer de aprender.

El alumnado había ingresado a la UNED de la siguiente forma (ver tabla 8 en anexos).

La distribución de la forma de acceso refleja que existe un alto porcentaje de alumnos que lo hace a través de selectividad, pero el mayor porcentaje accede mediante la realización del Curso de acceso para mayores de 25 años, lo que deja de manifiesto que la educación a distancia ofrece una segunda oportunidad a las personas que no pudieron estudiar en su juventud debido a sus circunstancias personales. También destaca que la educación superior a distancia es elegida por un amplio sector de titulados superiores para cursar una segunda o posterior carrera universitaria.

\section{Conclusiones}

En primer lugar, del análisis descriptivo realizado se puede concluir que las recomendaciones realizadas desde hace décadas por distintas instancias cobran más vigencia y valor en la actual situación de crisis económica que azota al mundo. La educación permanente ofrece una alternativa real a las personas que están desempleadas, pretenden mejorar o cambiar su trayectoria profesional, o simplemente deciden ocupar su tiempo de ocio en actividades formativas.

El imparable avance de las nuevas tecnologías ha posibilitado acercar y flexibilizar el acceso a la educación permanente a personas que por circunstancias laborales o familiares no pueden asistir a actividades educativas presenciales.

El significativo aumento de instituciones de educación a distancia y el incremento de la tasa de matriculación revelan que la educación superior a distancia constituye una alternativa muy valorada para realizar estudios universitarios en este país.

El paradigma educativo actual que preconiza el papel relevante del alumno frente a la institución, tiene su reflejo en las instituciones de educación superior a distancia, en las que el alumno decide los tiempos y ritmo de su actividad discente.

Asimismo, los datos analizados en el estudio experimental planteado revelan que las mujeres son mayoría en las aulas de las universidades a distancia, tal y como ocurre en las presenciales.

La mayoría del alumnado supera los treinta años, lo que confirma el carácter complementario de los centros de educación a distancia frente a los presenciales que albergan un alumnado de edad más joven, que puede asistir con mayor facilidad a clase al tener menos obligaciones laborales o familiares.

El alumnado encuestado en una amplia mayoría compagina sus estudios con la actividad laboral, este dato es muy significativo para confirmar que la educación a distancia ofrece mayores posibilidades a sus alumnos para conciliar su dedicación al estudio con la vida familiar y laboral.

La información recogida pone de manifiesto que la motivación para cursar estudios superiores responde a distintas necesidades, que van desde la promoción profesional, hasta el deseo de aprender por el placer de aprender o por realización personal; esta afirmación se desprende tanto por los datos referentes a la relación entre estudios cursados y la actividad laboral como, por las distintas modalidades de acceso del alumnado a los estudios universitarios.

A modo de conclusión final, se puede afirmar que la educación a distancia se ha constituido en una de las alternativas más eficaces y demandadas en el marco de la educación permanente. Junto a la educación presencial puede contribuir al proyecto de aprendizaje permanente de una persona. Las tecnologías de la información y la comunicación ofrecen múltiples 
BÚSQUEDA - Julio / Diciembre de 2015 - No. 15 (7 - 17)

y eficientes oportunidades para la enseñanza y para el aprendizaje permanente a distancia.

Asimismo, la educación permanente en cualquiera de sus modalidades requiere de docentes con competencias profesionales que les permitan adecuarse al perfil del alumnado y al proceso de enseñanza-aprendizaje que este paradigma exige. Sin duda, se plantea un reto muy importante para las instituciones responsables de la formación de los profesionales que deben cumplir con las expectativas que la sociedad demanda de la educación en la actualidad.

\section{Referencias bibliográficas}

Álvarez, L. (1998). Educación a Distancia. ¿Para qué y cómo? Recuperado de: http://www. sld.cu/libros/distancia/indice.html

Bajo, N. (2009). El principio revolucionario de la Educación Permanente. Anuario Jurídico y Económico Escurialense, XLII, 531-550.

Battenberg, R. W. (1971). The Boston Gazette, 20 de marzo, 1728. Epistolodidaktika, 1, pp. 44-45.

Caride, J.A., Lorenzo, J.J. y Rodríguez, M.A. (2012). Educar cotidianamente: el tiempo como escenario pedagógico y social en la adolescencia escolarizada. Pedagogía Social. Revista Interuniversitaria, 20, pp. 19-60.

Castells, M. (1997). La Era de la información: economía, sociedad y cultura. Vol. I: La sociedad red. Madrid: Alianza. En Sangrà Morer, A. (2002). Educación a distancia, educación presencial y usos de la tecnología: una tríada ara el progreso. Edutec. Revista Electrónica de Tecnología Educativa Núm. 1. /mayo 02. Recuperado de: http://www.uib.es/depart/gte/edutec-e/ revelec15/albert_sangra.htm

Chiecher A., Paoloni P. y Ficco, C (2014). Ingreso a la Universidad en modalidad a distancia. El papel de aspectos motivacionales y cognitivos en la configuración de logros académicos. RED, Revista de Educación a Distancia. Número 43. 15 de noviembre de 2014. Recuperado de: http://www.um.es/ $\mathrm{ead} / \mathrm{red} / 43$

Delors, J. (coord.) (1996). La Educación encierra un tesoro. Informe a la UNESCO de la Comisión Internacional sobre la Educación para el Siglo XXI. Madrid: Santillana Ediciones UNESCO.

García, L. (1986). Educación superior a distancia. Análisis de su eficacia. Badajoz: UNEDMérida.

García, L. (1990). Un concepto integrador de enseñanza a distancia. En Villarroel y Pereira. La educación a distancia: desarrollo y apertura. Caracas: ICDE.

García, L. (2002). La educación a distancia. De la teoría a la práctica. Barcelona: Ariel.

Garrison, D. R. (1985). Three generations of technological innovation in distance education, Distance Education, 6, pp. 235241.

Gimeno, J. (2008). El valor del tiempo en educación. Madrid: Morata. En Caride Gómez, J.A., Lorenzo Casteiras, J.J. y Rodríguez Fernández M.A. (2012). Educar cotidianamente: el tiempo como escenario pedagógico y social en la adolescencia escolarizada. Pedagogía Social. Revista Interuniversitaria, 20, pp. 19-60.

Graf, K. (1980). Correspondence instruction in the history of the western world, en Selected Papers on Distance Education. Hagen: Fernuniversität ZIFF.

Jorquera, C. (1999). La educación a distancia. Recuperado de: http://monografias.com/ trabajos/edudistancia/edudistancia.shtml

Martínez-Salanova, E. (2012). La educación permanente, un nuevo entorno pedagógico global. Recuperado de: 
http://www.uhu.es/cine.educacion/ didactica/0011educpermanente.htm

Mora, M. L. (2006). La educación a distancia una herramienta alterna para el desarrollo de los individuos durante toda la vida. Recuperado de: http://www.gestiopolis. com/canales6/eco/educacion-a-distanciay-el-desarrollo.htm

Padula, J. E. (2002). Educación a distancia. Una mirada sobre el alumno. Recuperado de: http://www.uned.es/catedraunesco-ead/ publicued/pbc02/art_bened1.htm

Pérez, R. (2006). Evaluación de programas educativos. Madrid: Editorial La Muralla.

Resolución del Consejo de la Unión Europea de 27 de junio de 2002 sobre la educación permanente. Publicada en el Diario Oficial de las Comunidades Europeas el 9 de julio de 2002.

Sangrà, A. (2002). Educación a distancia, educación presencial y usos de la tecnología: una tríada ara el progreso. Edutec. Revista Electrónica de Tecnología Educativa Núm. 1. /mayo 02. Recuperado de: http://www.uib.es/depart/gte/edutec-e/ revelec15/albert_sangra.htm
Sarramona, J. (2001). Evaluación de programas de educación a distancia. Revista Iberoamericana de Educación a Distancia (RIED). Vol.4. núm. 1. pp. 9-37.

Taylor, J. C. (1995). Distance education technologies: The fourth generation. Australian Journal of Educational Technology, 11, 2.

Taylor, J. C. (1999). Distance education. The fifth generation, XIX World Conference ICDE'1999. Viena: ICDE.

UNESCO (2015). Documento de posición sobre la educación después de 2015. Recuperado de: http://unesdoc.unesco. org/images/0022/002273/227336s. pdf

XIX Reunión de la Organización de las Naciones Unidas para la Educación, la Ciencia y la Cultura celebrada en Nairobi, 26 de octubre - 30 de noviembre de 1976. 